\title{
Acceptance and commitment therapy as a part of a multimodal rehabilitation for patients with schizophrenia
}

\begin{abstract}
Schizophrenia is a chronic mental disorder. The development of efficacious and effective psychosocial treatments is imperative for the treatment of people with schizophrenia. The main therapy for those people is the pharmacotherapy. Cognitive Behaviour Therapy (CBT) and various rehabilitation programs present an effective and efficacious therapy in combination with pharmacotherapy for individuals with schizophrenia. Acceptance and Commitment Therapy belongs to the third wave of behavioural therapies. This mini review will focus on the latest evidenced based data regarding the efficacy of Acceptance and Commitment Therapy in people with schizophrenia. In addition, the possible combination of ACT with other evidenced based psychological interventions will be addressed. It seems to be that ACT is an effective therapeutic approach. Further meta analyses and randomized controlled trials regarding the efficacy and effectiveness of ACT in individuals with schizophrenia should be conducted. There are some interesting clinical and research questions, which will be discussed. ACT should be recommended as an alternative and adjunct therapy in combination with pharmacotherapy, Cognitive Behavioural Therapy, behavioural family therapy and other efficacious rehabilitation programs. ACT should be adapted as a part of a multimodal rehabilitation for people with schizophrenia. Further research regarding the efficacy and effectiveness of ACT in longitudinal studies with large samples in combination with other evidenced based interventions in Cognitive Behaviour Therapy is recommended.
\end{abstract}

Keywords: schizophrenia, acceptance and commitment therapy (act), cognitive behavioural therapy (cbt), evidenced based interventions
Volume 3 Issue 5 - 2015

\author{
Stavroula Rakitzi,' Polyxeni Georgila² \\ 'Diplom Psychologist (Msc Clinical Psychology) and Cognitive \\ behavioral Psychotherapist, Greece \\ ${ }^{2}$ Psychiatrist, Director of the psychiatric department for adults \\ of the General Hospital “G. Gennimatas”, Greece
}

Correspondence: Stavroula Rakitzi, Diplom Psychologist (Msc Clinical Psychology) and Cognitive behavioral Psychotherapist, Greece, Tel 210-3824669, 6936736473, Fax 210-3824669, Email srakitzi@gmail.com

Received: July 15, 2015 | Published: September 23, 2015

\section{Introduction}

Schizophrenia is a chronic and debilitating condition. Patients with schizophrenia experience positive, negative symptoms, cognitive dysfunctions and other mental health problems as comorbidity. The suicidality risk of people with schizophrenia is very high. When the positive and negative symptoms are not treated properly, the risk for a hospitalization is very high.

The main therapy for patients with schizophrenia is the pharmacotherapy. Cognitive Behaviour Therapy (CBT) presents an effective and efficacious therapy and it is recommended as an adjunctive treatment in combination with pharmacotherapy. ${ }^{1}$ There are also many effective and efficacious rehabilitation programs for people with schizophrenia, who are available in the community. ${ }^{2}$

Cognitive behavioural therapy has been found to be efficacious in the treatment of schizophrenia. ${ }^{3-12}$ Acceptance and Commitment Therapy has been featured as one of the third generation of behavioural therapies. The first generation was the classic behaviour therapy and the second generation was the cognitive therapy. ACT proposes the following strategies: Acceptance, defusion, present moment focus, self as perspective, values and committed action. The four core processes of acceptance, defusion, self as perspective and contact with the present moment are conceptualized as mindfulness and acceptance processes. Those of self as perspective, contact with the present moment, values and committed action are conceptualized as commitment and behaviour change processes. ${ }^{13}$

This review will focus on the latest evidenced based data regarding the efficacy of Acceptance and Commitment Therapy in people with schizophrenia. In addition, the possible combination of ACT with other evidenced based psychological interventions will be addressed.

\section{Discussion}

Cognitive behavioural therapy has been found to be efficacious in the treatment of schizophrenia. ${ }^{3-12}$ A meta analysis includes 60 RCTs (4234 participants) on psychiatric disorders, somatic disorders and stress at work. The mean effect size across all comparisons was small (0. 42). Wenn ACT was compared to various forms of cognitive or behavioural treatments a small and non-significant effect size of 0 . 16 was obtained. ACT is probably efficacious for chronic pain and tinnitus, possible efficacious for depression, psychotic symptoms, OCD, mixed anxiety, drug abuse and stress at work. An evidenced base evaluation showed that ACT is not yet well-established for any disorder. ${ }^{14}$

A meta-analysis of 39 randomized controlled trials on the efficacy of ACT with 1.821 patients with mental disorders or somatic health problems highlighted the following results: The ACT Group had better outcomes in comparison to waitlist $(\mathrm{g}=0.82)$, to psychological placebo ( $\mathrm{g}=0.51)$ and to treatment as usual (TAU) $(\mathrm{g}=0.64)$. ACT was better on secondary outcomes $(g=0.30)$, life satisfaction/quality measures $(\mathrm{g}=0.37)$ and process measures $(\mathrm{g}=0.56)$ in comparison to control conditions. There were no significant differences between ACT and established treatments (cognitive behavioural therapy). ${ }^{15}$

Therefore, ACT is better than treatment as usual or placebo and has shown the same effectiveness in treating anxiety disorders, depression, addiction and somatic health problems with established psychological interventions. ${ }^{15}$ 
Psychiatric inpatients with psychotic symptoms were randomized to two groups: Treatment as usual and treatment as usual plus individual sessions of ACT. The ACT Group had better outcomes in affective symptoms, overall improvement, social impairment and distress as a result of the hallucinations. More participants of the ACT group showed clinically significant symptom improvement at discharge. The coping strategies with the hallucinations was better in the ACT group. ${ }^{16}$

CBT leads to the modification of dysfunctional thought and ACT leads to the modification of the relationship between the person and its own thinking through mindfulness and acceptance. Therefore, it could be easier for patients with psychosis to develop new strategies for coping with psychotic symptoms using ACT than more traditional therapies, at least in the short term. Finally, ACT could be implemented in combination with the behavioural family therapy and cognitivebehavioural therapies. ${ }^{16}$

This study supports the effectiveness of ACT. Further studies with larger samples will be appropriate, in order to characterize ACT as a standard treatment for patients with schizophrenia. Nevertheless, ACT can easily implemented in acute-treatment settings. ${ }^{16}$

Both the early trials of ACT for psychosis ${ }^{16,17}$ were designed as "proof of concept" studies with extraordinarily brief treatment input (four and three sessions, respectively) and limited outcome targets. The following study focused on hospitalization alone and extended the results of Bach \& Hayes. ${ }^{17}$ As compared to TAU, participation in ACT was associated with reduced rehospitalisation at 1 year post discharge for inpatients with psychotic disorders and mood disorders with psychotic features after controlling the length of previous and current hospitalization. What the present study adds is evidence that some of the effects of these early pilot studies of ACT for psychosis may be relatively long-lasting. ACT could be included in such broad packages, especially if its core messages suggesting that cognitive defusion may be a particularly important pathway of change in ACT for psychosis. ${ }^{18}$

It seems to be that ACT is an effective therapeutic approach. Further meta analyses and randomized controlled trials regarding the efficacy and effectiveness of ACT in individuals with schizophrenia should be conducted.

Whereas traditional CBT focuses on directly modifying dysfunctional thought content through cognitive restructuring, ACT focuses on modifying the person's relationship to his or her thinking through mindfulness and acceptance. ${ }^{16}$

There are some interesting clinical and research questions, which have to be addressed:

a. How many sessions are recommended for ACT in individuals with schizophrenia?

b. Can ACT be implemented before, after or during the implementation of an evidenced based cognitive behavioural approach?

c. What is the impact of ACT on the improvement of therapeutic alliance with people with schizophrenia?

d. What is the impact of the implementation of ACT on the metacogntive ability and insight of individuals with schizophrenia?

\section{Conclusion}

Acceptance and Commitment Therapy belongs to the third wave of behavioural therapies. ACT is a promising therapeutic approach in combination with interventions of Cognitive behavioural Therapy and other evidenced based rehabilitation programs for individuals with schizophrenia. ACT should be recommended as an alternative and adjunct therapy in combination with pharmacotherapy, Cognitive Behavioural Therapy, behavioural family therapy and other rehabilitation programs. ACT should be adapted as a part of a multimodal rehabilitation for people with schizophrenia. It could be recommended to research the efficacy and effectiveness of ACT in longitudinal studies with large samples in combination with other evidenced based interventions for people with schizophrenia.

\section{Acknowledgments}

None.

\section{Conflicts of interest}

Author declares there are no conflicts of interest.

\section{Funding}

None

\section{References}

1. National Institute for Health and Care Excellence (2014) Psychosis and schizophrenia in adults: treatment and management. NICE clinical guideline 178. NICE.

2. Roder V, Medalia A (2010) Neurocognition and social cognition in schizophrenia patients. Basic concepts and treatment. Karger, Basel, Switzerland.

3. Turkington D, Dudley R, Warman DM, Beck AT (2006) Cognitive Behavioural Therapy for schizophrenia: A Review. Focus 4(2): 223-233.

4. Wykes T, Steel C, Everitt B, Tarrier N (2008) Cognitive behaviour therapy for schizophrenia: effect sizes, clinical models and methodological rigor Schizophr Bull34(3): 523-537.

5. Wykes T, Spaulding WD (2011) Thinking about the future Cognitive Remediation Therapy-What works and could we do better? Schizophr Bull 37(Suppl 2): S80-S90.

6. Freeman D (2011) Improving cognitive treatment for delusions, Schizophr Res 132(2-3): 135-139.

7. Wunderlich U, Wiedemann G, Buchkremer G (1996) Sind psychosoziale Interventionen bei schizophrenen Patienten wirksam? Eine Metaanalyse. Verhaltenstherapie 6: 4-13.

8. Rector NA, Beck AT (2001) Cognitive Behavioral Therapy for schizophrenia: An empirical Review. J Nerv Ment Dis 189(5): 278-287.

9. Pfammatter M, Junghan UM, Brenner HD (2006) Efficacy of psychological Therapy in Schizophrenia: Conclusions from Metaanalyses. Schizophr Bull 32 (Suppl 1): S64-S80.

10. Mojtabai R, Nicholson RA, Carpenter BN (1998) Role of psychosocial treatment in management of schizophrenia: a meta analytic review of controlled outcome studies. Schizophr Bull 24(4): 569-587.

11. Gould RA, Mueser KT, Bolton E, Mays V, Goff D (2001) Cognitive Therapy for psychosis in schizophrenia: an effect size analysis. Schizophr Res 48: 335-342.

12. Grant PM, Huh GA, Perivoliotis D, Stolar NM, Beck, AT (2012) Randomized Trial to Evaluate the Efficacy of Cognitive Therapy for Low-Functioning Patients With Schizophrenia. Arch Gen Psychiatry 69(2): 121-127.

13. Waltz TJ, Hayes SC (2010) Acceptance and Commitment Therapy. In Kazantzis N, et al. (Eds.), Cognitive and behavioural theories in clinical practice, The Guilford Press, New York, USA, pp. 148-192. 
14. Ost LG (2014) The efficacy of Acceptance and Commitment Therapy: an updated systematic review and meta-analysis. Behav Res Ther 61: 105-121.

15. A-Tiak JG, Davis ML, Morina N, Powers MB, Smits JA, et al. (2015) A meta-analysis of the efficacy of acceptance and commitment therapy for clinically relevant mental and physical health problems. Psychother Psychosom 84(1): 30-36.

16. Gaudiano BA, Herbert JD (2006) Acute treatment of inpatients with psychotic symptoms using Acceptance and Commitment Therapy: Pilot results. Beh Res Therapy 44(3): 415-437.
17. Bach P, Hayes SC (2002) The use of Acceptance and Commitment Therapy to prevent the rehospitalization of psychotic patients: A randomized controlled trial. J Cons Clin Psychology 70(5): 1129-1139.

18. Bach P, Hayes SC, Gallop R (2012) Long term effects of brief acceptance and commitment therapy for psychosis. Behav Modif 36(2): 165-181. 